\title{
Numerical solution for diffusion equations with distributed order in time using a Chebyshev collocation method
}

\author{
Maria Luísa Morgado ${ }^{\mathrm{a}, *}$, Magda Rebelo ${ }^{\mathrm{b}}$, Luis L. Ferrás ${ }^{\mathrm{c}, \mathrm{d}}$, Neville Ford ${ }^{\mathrm{d}}$ \\ ${ }^{a}$ Centro de Matemática, pólo CMAT-UTAD, Departamento de Matemática, Universidade de Trás-os-Montes e Alto \\ Douro, UTAD, Quinta de Prados 5001-801, Vila Real, Portugal \\ ${ }^{b}$ Centro de Matemática e Aplicações (CMA) and Departamento de Matemática, Faculdade de Ciências e \\ Tecnologia, Universidade NOVA de Lisboa, Quinta da Torre, 2829-516 Caparica, Portugal \\ ${ }^{c}$ Institute for Polymers and Composites, University of Minho, Campus de Azurém 4800-058 Guimarães, Portugal \\ ${ }^{d}$ Department of Mathematics, University of Chester, CH1 4BJ, UK
}

\begin{abstract}
In this work we present a new numerical method for the solution of the distributed order timefractional diffusion equation. The method is based on the approximation of the solution by a double Chebyshev truncated series, and the subsequent collocation of the resulting discretised system of equations at suitable collocation points. An error analysis is provided and a comparison with other methods used in the solution of this type of equation is also performed.
\end{abstract}

Keywords: Fractional differential equation, Caputo derivative, Diffusion equation, Chebyshev polynomials, Spectral methods, Distributed order equation.

2000 MSC: 26A33, 41A50

\section{Introduction}

In 1827, the botanist Robert Brown observed the intriguing jittering movement of small particles such as pollen grains, when these were immersed in water. Nowadays it is well known that this motion is caused by the rapid movement of water molecules, and insight into this problem was provided by Albert Einstein in 1905, in his work regarding Brownian motion, entitled "On the motion, required by the molecular-kinetic theory of heat, of particles suspended in fluids at rest." [22]. His work served as a definitive confirmation that atoms and molecules actually exist. Although molecules are too small to be seen directly, their presence can be inferred from their visible effect on larger grains (such as the pollen grains). In his doctoral dissertation, Einstein developed a statistical molecular theory of liquids and in his subsequent paper he took the view that Brownian motion could be explained in terms of a type of stochastic process called a "random walk" (it is worth mentioning that Louis Bachelier, a student of Henri Poincaré, developed a theory of Brownian motion in his 1900 thesis [1] regarding stock market fluctuation [39]).

The random walk theory was popularised by Karl Pearson in his letter to Nature (1905) [55], where he proposed the following problem: a man starts from a point $O$ and walks $l$ yards in a

\footnotetext{
${ }^{*}$ Corresponding author

Email addresses: luisam@utad.pt (Maria Luísa Morgado ), msjr@fct.unl.pt (Magda Rebelo), luis.ferras@dep.uminho.pt (Luis L. Ferrás ), njford@chester.ac.uk (Neville Ford)
} 
straight line; after this step he turns through a random angle and takes another step of length $l$. He repeats this process $n$ times. What is the probability that after $n$ steps he is at a distance between $r$ and $r+\delta r$ from the original point $O$ ? This problem is basically the idea behind Brownian motion, but the connection between the diffusion law proposed by Einstein,

$$
\frac{\partial u(x, t)}{\partial t}=D \frac{\partial^{2} u(x, t)}{\partial x^{2}}
$$

and the random walk process, was due to the excellent work of M. von Smoluchowski [61] in 1906.

In Brownian motion theory, the mean squared displacement, $\left\langle x^{2}\right\rangle$, of a particle is proportional to the time $t,\left\langle x^{2}\right\rangle=2 D t$, where $D$ is the diffusion coefficient and $\left\langle x^{2}\right\rangle$ is the second moment of the Gaussian distribution that governs the probability of being at position $x$ at time $t$. This Gaussian distribution was observed experimentally, for example in the work of Kappler [37], and, if we track the diffusion of a particle that follows the governing equation given by equation (1) and is at the origin at time $t=0$, then we obtain the solution as the normalised Gauss probability density function,

$$
u(x, t)=\frac{1}{\sqrt{4 \pi D t}} \exp \left(-\frac{x^{2}}{4 D t}\right) .
$$

Although this linear growth of the mean squared displacement is observed in most diffusion problems, there is a considerable number of physical processes that do not show this behaviour $([4,51,50,32,52])$, but present an anomalous diffusion, that can be subdiffusive or supperdifusive. Note that, by 1926, Lewis Fry Richardson [56] studied the relative diffusion of two tracer particles in a turbulent flow and found that $\left\langle x^{2}\right\rangle \simeq t^{3}$, meaning that a supperdifusive behaviour was obtained.

In general, anomalous diffusion refers to the following power-law form,

$$
<x^{2}>\simeq D_{\alpha} t^{\alpha}
$$

where we have subdiffusion for $0<\alpha<1$ and superdiffusion for $\alpha>1$.

We may question how we can obtain a governing equation for a diffusion process that behaves like equation (3). The first idea is to take a look at the physical process under examination and find a suitable stochastic model. The problem is that we may find different models for different problems, leading to the difficult task of finding a universal theory for such non-universal and process dependent behaviour.

In order to understand the variety of possibilities, we can take, for example, the continuous time random walk process (CTRW) $([53,59])$, that generalises the random walk proposed by Pearson. In the CTRW we may have jumps of different length and a particle may wait before the next jump. Different distributions may be used for the waiting time and jump length and, therefore, different regimes of diffusion may be obtained, allowing in this way the development of a general theory that can be tuned according to the characteristics of the physical process.

For example, in the particular case of Lévy flights $[47,23,34,64,26]$ there is no characteristic size for the random walk jumps, and therefore, the variance or second moment is infinite, and does not follow a law such as the one given by equation (3). This does not mean that the Lévy flights are not important for modelling real world diffusion phenomena. In fact they find a wide range of applications including chaotic phase diffusion in Josephson junctions, turbulent diffusion, micelle 
dynamics, vortex dynamics, anomalous diffusion in rotating flows, etc [39]. It is all just a question of definition of the mean squared displacement. If we ask for the mean squared length of a jump then the answer is infinity, but, if instead we try to understand the properties of the distribution used in the Lévy flights by asking how far a Lévy walk has travelled from its starting point at time $t$, then the answer would be a time-dependent moment of the probability distribution [39].

As a second example $[52,15,60,58]$, let us consider a finite variance of the jump length and the wait time assumed to be given by a power-law distribution $\varphi(t)=\tau^{\alpha} / t^{1+\alpha}(0<\alpha<1)$ that leads to a divergent mean waiting time. A process modelled in this way leads to subdiffusion with a mean squared displacement given by $\left.<x^{2}\right\rangle=\left(2 K_{\alpha} / \Gamma(1+\alpha)\right) t^{\alpha}$. The governing equation, that tells us the probability of finding a particle at position $x$ at time $t$, is given by

$$
\frac{\partial^{\alpha} u(x, t)}{\partial t^{\alpha}}=K_{\alpha} \frac{\partial^{2} u(x, t)}{\partial x^{2}}
$$

where the fractional derivative on the left-hand-side of the equation is of Caputo type $([19,58])$ :

$$
\frac{\partial^{\alpha} u(x, t)}{\partial t^{\alpha}}=\frac{1}{\Gamma(1-\alpha)} \int_{0}^{t}(t-s)^{-\alpha} \frac{\partial^{n} u(x, s)}{\partial s^{n}} d s,
$$

with $n$ being the smallest integer greater than or equal to $\alpha$. This equation is known as a timefractional diffusion equation (TFDE) and has found application in a broad variety of engineering, biological, finance and physical processes where anomalous diffusion occurs (see, for example [31], [46] and [52]).

Regarding fractional differential calculus, it is wrong to think that this is a recent subject. For instance, the symbol $d^{n} y / d x^{n}$ was first proposed by Leibniz, and, in 1695 L'Hôpital asked Leibniz the meaning of $d^{n} y / d x^{n}$ if $n=1 / 2$. Several well known mathematicians tried to develop this general theory. In 1697, Leibniz sent letters to J. Wallis and J. Bernoulli, and, he mentioned the possible approach to fractional-order differentiation. In 1716 Leibniz died, but the urge to understand the derivatives of fractional order increased, and several other authors devoted their time to the subject. The well known Leonard Euler also contributed to the understanding and generalization of fractional differential calculus. He generalised the notion of factorial $n$ ! to non-integer values, called the gamma function $(\Gamma()$.$) , by Adrien-Marie Legendre around 1811. Later, Joseph Fourier$ (1768-1830) gave the first step to the generalisation of the notion of differentiation for arbitrary functions with his 1822 book, Theorie Analytique de la Chaleur. In 1832, Liouville presented two different definitions for the fractional derivative. Riemann also contributed to this subject and the ideas of Riemann and Liouville, led to the Riemann-Liouville definition of fractional integrals and derivatives. Grünwald (1867) and Post (1930) presented the idea of fractional derivative as the limit of a sum, using the classical definitions of a derivative. In 1927, Marchaud formulated an equivalent fractional derivative of arbitrary order $\alpha$ (with $0<\alpha<1$ ). In 1940, Erdélyi and Kober presented what we call now the Erdélyi-Kober fractional integral. This operator generalises the Riemann fractional integral and the Weyl integral. Later, Riesz also proposed a fractional integral of order $\alpha$, and in 1967 Caputo developed the Caputo fractional derivative presented in equation (5). Several other works can be found in the literature that contributed to the development of fractional calculus and the interested reader should consult [57]. It remains impossible to say that one definition is better than the other one (although it is fair to say that the Caputo definition is most often used for the application of fractional calculus to physics).

Returning to equation (4), it is worth mentioning that in recent decades great attention has 
been devoted to the general form of equation (4) given by,

$$
\frac{\partial^{\alpha} u(x, t)}{\partial t^{\alpha}}=D_{\alpha} \frac{\partial^{2} u(x, t)}{\partial x^{2}}+f(x, t), \quad 0<t \leq a, 0<x<b,
$$

where $D_{\alpha}$ stands for a general diffusion coefficient with dimensions $[\text { length }]^{2} /[\text { time }]^{\alpha}$.

Equation (6) is then useful for diffusion processes that follow (3) but, in the real world, there are more complex systems that do not follow equation (3) and that may present more than one characteristic scale. For example we saw that in the Lévy flights there is an absence of a characteristic scale. This means that fractional differential equations are a good tool for modelling some of the processes, but, in order to model correctly more complex systems a more powerful mathematical tool should be used.

The answer to this problem of non-unique scalability was given by the creation of distributed order fractional differential equations $[9,10]$ that proved to be useful in modelling anomalous diffusion characterised by two or more scaling exponents in the mean squared displacement (equation (3)), more precisely, in modelling processes for which the diffusion exponent can change in the course of time, as is the case of complex systems where the morphology of the material changes along the process. A close look into the physics of some complex diffusive processes, suggests that an even more general theory for fractional derivatives should be devised, and, this will no doubt be developed in the near future $([58,50,52,39])$.

As Chechkin, Gorenflo and Sokolov [9] observed, "the development of numerical schemes for solving distributed-order kinetic equations and for modelling sample paths of the random processes governed by these equations is also of importance.", meaning that numerical schemes for the solution of this type of equation are demanded. This is the main motivation for the present work, where we present a numerical method for the solution of the general distributed-order time fractional diffusion equation,

$$
\int_{0}^{1} c(\alpha) \frac{\partial^{\alpha} u(x, t)}{\partial t^{\alpha}} d \alpha=\frac{\partial^{2} u(x, t)}{\partial x^{2}}+f(x, t), \quad 0<t \leq a, 0<x<b,
$$

where the function $c(\alpha)$ acting as weight for the order of differentiation is such that ([30], [45]) $c(\alpha) \geq 0$ and $\int_{0}^{1} c(\alpha) d \alpha=C>0$. Obviously, if $c(\beta)=\frac{\delta(\alpha)}{D^{\alpha}}$, where $\delta()$ is the delta Dirac function, then (7) reduces to (6). Note that the dimensions of $c(\alpha)$ are $[\text { time }]^{\alpha} /[\text { length }]^{2}$. For an alternative to these dimensions please consult the work of Chechkin et al. [10] where they use an extra constant that represents a characteristic time of the problem.

Here, we will be interested in the numerical approximation of this type of distributed-order equation with boundary conditions of Dirichlet type:

$$
u(0, t)=\phi_{0}(t), u(b, t)=\phi_{b}(t), \quad 0<t<a,
$$

and initial condition

$$
u(x, 0)=g_{0}(x), \quad 0<x<b .
$$

The concept of distributed order was developed by Caputo [6] and further developed by Caputo, 
Bagley and Torvik $[7,8,2,3]$.

With the growing interest on this type of equation, numerical methods started being developed, for example in the work by Diethelm and Ford [16] where they present a basic framework for the numerical solution of distributed order differential equations (see also [17, 18]). A more recent increase in interest in the use of distributed order differential equations (particularly in the case where the derivatives are given in the Caputo sense) led Ford and Morgado [24] to discuss the existence and uniqueness of solutions for this type of equation, and also to propose a numerical method for their approximation in the case where the initial conditions are not known (with boundary conditions being given away from the origin). Two years later, Katsikadelis [38] devised a numerical method for the solution of the distributed order FDE approximating them with a multiterm FDE (that is solved by adjusting appropriately the numerical method developed for multi-term FDEs). In the same year, Liao et al. [43] investigated a class of modified Du FortFrankel-type schemes for fractional subdiffusion equations in the Jumaries modified Riemanniouville form with constant, variable or distributed fractional order.

In 2015, some papers were published on the numerical solution of distributed order FDEs. Morgado and Rebelo [54] presented an implicit scheme for the numerical approximation of the distributed order time-fractional reaction-diffusion equation with a nonlinear source term (see also [25]), Ye et al. presented two papers, one [65] considering the time distributed-order and Riesz space fractional diffusion on bounded domains with Dirichlet boundary conditions (deriving an implicit difference method for the multi-term time-space fractional diffusion equation) and the other [66] presenting a numerical method based on a compact difference scheme for a distributed order time-fractional diffusion-wave equation. $\mathrm{Hu}$ et al. [33] considered a new time distributed-order and two-sided space-fractional advection-dispersion equation that was solved numerically using an implicit method for the solution the multi-term fractional equation. Gao et al. published a series of papers [27, 28, 29] where: [27] two difference schemes were derived for both one-dimensional and two-dimensional distributed-order differential equations (he proved that the schemes are unconditionally stable and convergent); [28] the Grünwald formula was used to solve the one-dimensional distributed-order differential equations (two difference schemes were derived and the extrapolation method was applied to improve the approximate accuracy); [29] two alternating direction implicit difference schemes were derived for two-dimensional distributed-order fractional diffusion equations (he proved that the schemes are unconditionally stable and convergent). Wang et al. [63] derived and analysed a second-order accurate implicit numerical method for the Riesz space distributedorder advection-dispersion equation. Duong et al. [21] proposed a novel numerical scheme for analysing the behaviour of a distributed order linear single input single output control system under random forcing. The method is based on the operational matrix technique to handle stochastic distributed order systems. Jin et al. [35] presented a numerical solution of an initial boundary value problem for the distributed order time fractional diffusion equation. They developed a space semidiscrete scheme based on the standard Galerkin finite element method, and established error estimates for both smooth and nonsmooth initial data.

Finally, Chen et al. [14] developed a mixed finite difference/spectral method, and, Li et al. [41] proposed a numerical method for solving distributed order diffusion equations by using a classical numerical quadrature formula, and the resulting multi-term time-fractional diffusion equation were solved by the reproducing kernel method.

In almost all the methods described so far, only finite difference approximations have been considered for the fractional time derivative, and these methods may become computationally 
heavy, due to the non-local property of fractional differential operators. Many of these authors assumed certain regularity assumptions on the solution in order to provide the convergence analysis of their numerical schemes, although it is widely known that the solution of fractional differential equations may be nonsmooth at $t=0$ even if the data is infinitely smooth. However, as Diethelm and Ford pointed out in [18], under certain simple hypotheses, solutions of the distributed order equations can be shown to be smooth. We have adapted their analysis (to be published in a sequel) for the distributed order diffusion equation and this provides a theoretical basis for our convergence analysis in this paper.

On the other hand, the idea of approximating the solution of fractional differential equations with truncated Chebyshev series has been widely exploited (see, for example, [36, 20] and the references cited therein), but to the best of our knowledge, a complete and detailed error analysis has not yet been provided.

Hence, this paper has two purposes: the first one is to develop a numerical scheme with a lower computational cost, based on the approximation of the solution with a double truncated Chebyshev series, and the subsequent collocation of the resulting discretised system of equations at suitable collocation points; the second one is to provide an error analysis for such methods, which, as far as we are aware, has not previously been presented for fractional differential equations.

The paper is organised as follows. After this Introduction, we present, in Section 2 some preliminaries results that will be useful in the derivation of the numerical method, which will be described in Section 3. In Section 4 we provide a convergence analysis of the numerical scheme. In Section 5 we test the numerical method considering different examples and we compare them with previous ones. The paper ends with the conclusions in Section 6 .

\section{Preliminaries}

We begin this section with some results on the well-posedness of the problem we intend to solve numerically. Such results are still very scarce and may be resumed in the following theorems (adapted from [44]).

First, we define the spaces

$$
\begin{aligned}
& W_{t}^{1}((0, a))=\left\{g \in C^{1}((0, a]) \text { such that } g^{\prime} \in L((0, a))\right\} . \\
& M_{\Delta}=\left\{g \in C_{t}^{1}([0, a]) \cap C_{x}^{2}(0, b) \text { such that } \frac{\partial^{2} g}{\partial x^{2}} \in L^{2}((0, b)) \text { and } g(0, t)=g(b, t)=0\right\} .
\end{aligned}
$$

Theorem 2.1. Consider the distributed-order time fractional diffusion equation (7) with boundary conditions of Dirichlet type (8) and initial condition (9).

If $f(x, t) \in C((0, b) \times(0, a)), \phi_{0}(t), \phi_{b}(t) \in C([0, a])$ and $g_{0}(x) \in C([0, b])$, then the problem (7)-(9) possesses at most one solution $u(x, t) \in C_{t}([0, a]) \cap W_{t}^{1}((0, a)) \cap C_{x}^{2}((0, b))$. Moreover, that solution, if it exists, continuously depends on the data given in the problem as follows

$$
\|u-\tilde{u}\|_{\infty} \leq \max \left\{\epsilon_{0}, \epsilon_{1}, \epsilon_{2}\right\}+\frac{a^{\alpha}}{C \Gamma(1+\alpha)} \epsilon,
$$

for the solutions $u$ and $\tilde{u}$ of the problem (7)-(9) with the data $f, g_{0}, \phi_{0}, \phi_{b}$ and $\tilde{f}, \tilde{g}_{0}, \tilde{\phi}_{0}, \tilde{\phi}_{b}$, respectively, that satisfy the conditions $\|f-\tilde{f}\|_{\infty} \leq \epsilon,\left\|g_{0}-\tilde{g}_{0}\right\|_{\infty} \leq \epsilon_{0},\left\|\phi_{0}-\tilde{\phi}_{0}\right\|_{\infty} \leq \epsilon_{1}$ and $\left\|\phi_{b}-\tilde{\phi}_{b}\right\|_{\infty} \leq \epsilon_{2}$. 
In [44], Luchko also addressed the question of existence of solution. He considered the case where $\phi_{0}(x)=\phi_{b}(x) \equiv 0$ (mentioning that the more general case of non-vanishing functions $\phi$ could be reduced to this one) and he stated sufficient conditions for the existence of a formal solution, which could be regarded as a classical solution of the problem, that is, a function $u \in$ $C_{t}([0, a]) \cap W_{t}^{1}((0, a)) \cap C_{x}^{2}((0, b))$ satisfying the differential equation together with the initial and bondary conditions.

Theorem 2.2. If the conditions of Theorem 2.1 are satisfied and if the source function $f \in M_{\Delta}$ and $g_{0} \in M_{\Delta}$, then there exists a solution $u(x, t)$ of the problem (7)-(9) that belongs to the space $C_{t}([0, a]) \cap W_{t}^{1}((0, a)) \cap C_{x}^{2}((0, b))$.

Next, we present some auxiliary results that will be used in the derivation of our proposed numerical scheme, which is based on the representation of the solution by a truncated Chebyshev series expansion. Hence, we begin this section with some results related to this approach.

Chebyshev polynomials of degree $n, T_{n}(z)=\cos (n \arccos (z))$, are defined on the interval $[-1,1]$. In order to use them on the interval $[0, L]$, for any real $L>0$, we introduce the change of variable $z=2 t / L-1$ and obtain the so-called shifted Chebyshev polynomials $T_{L, n}(t)=T_{n}\left(\frac{2 t}{L}-1\right)$. These shifted Chebyshev polynomials can also be obtained from the following expression (see [20]):

$$
T_{L, n}(t)=n \sum_{k=0}^{n}(-1)^{n-k} \frac{2^{2 k}(n+k-1) !}{(2 k) !(n-k) ! L^{k}} t^{k}, \quad n=1,2, \ldots,
$$

where

$$
T_{L, i}(0)=(-1)^{i} \text { and } T_{L, i}(L)=1,
$$

and satisfy the following orthogonality relation:

$$
\int_{0}^{L} T_{L, j}(t) T_{L, k}(t) \omega_{L}(t) d t=\delta_{k j} h_{k}
$$

with $\omega_{L}(t)=\frac{1}{\sqrt{L t-t^{2}}}$ and $h_{0}=\pi, h_{k}=\frac{\pi}{2}, k=1,2, \ldots$.

The Chebyshev series expansion of a function $f(x, t),(x, t) \in[-1,1] \times[-1,1]$, can be given by

$$
f(x, t)=\sum_{i=0}^{\infty} \sum_{j=0}^{\infty} a_{i j} T_{i}(t) T_{j}(x)=\sum_{i=0}^{\infty} \sum_{j=0}^{\infty} a_{i j} T_{j i}(x, t),
$$

where $T_{j i}(x, t)=T_{i}(t) T_{j}(x)$. In Mason [48] the proof of convergence of the series is provided, under the assumption that $f(x, t)$ is of bounded variation and that one of the partial derivatives is bounded (see also the works of Chen et al. [11, 12, 13]).

Generalising $(13)$ for $(x, t) \in[0, b] \times[0, a]$ the series expansion is given by:

$$
f(x, t)=\sum_{i=0}^{\infty} \sum_{j=0}^{\infty} a_{i j} T_{a, i}(t) T_{b, j}(x)=\sum_{i=0}^{\infty} \sum_{j=0}^{\infty} a_{i j} T_{j i}^{(b, a)}(x, t)(t),
$$


where $T_{a, i}(t)=T_{i}\left(\frac{2 t}{a}-1\right), T_{b, j}(x)=T_{j}\left(\frac{2 x}{b}-1\right)$ and $T_{j i}^{(b, a)}(x, t)=T_{a, i}(t) T_{b, j}(x)$.

The coefficients $a_{i j}$ are given by

$$
a_{i j}=\frac{e_{i} e_{j}\left\langle f(x, t), T_{j i}^{(b, a)}(x, t)\right\rangle}{\pi^{2}}=\frac{e_{i} e_{j}}{\pi^{2}} \int_{0}^{a} \int_{0}^{b} \frac{f(x, t) T_{a, i}(t) T_{b, j}(x)}{\sqrt{a t-t^{2}} \sqrt{b x-x^{2}}} d x d t, \quad i, j=0,1,2, \ldots
$$

where

$$
e_{i}= \begin{cases}1, & i=0 \\ 2, & i>0\end{cases}
$$

For computational purposes, only the first $(n+1)$ and $(m+1)$ terms of the series are considered, meaning that a function $f(x, t)$ of two independent variables, $(x, t) \in[-1,1] \times[-1,1]$, may be approximately expanded in terms of truncated double Chebyshev series

$$
P_{n, m}(f)(x, t) \equiv f_{n, m}(x, t)=\sum_{i=0}^{n} \sum_{j=0}^{m} a_{i j} T_{i}(t) T_{j}(x)
$$

or

$$
P_{n, m}(f)(x, t) \equiv f_{n, m}(x, t)=\sum_{i=0}^{n} \sum_{j=0}^{m} a_{i j} T_{a, i}(t) T_{b, j}(x),
$$

if $(x, t) \in[0, b] \times[0, a]$.

In the work of Sommariva et al. [62], it is stated that if $f$ is continuous and of bounded variation, then for every $\varepsilon>0$, there exists an $n(\varepsilon)$, and a sequence $m_{k}=m(k ; \varepsilon)$, such that $\left\|f-P_{n(\epsilon), m_{k}}\right\|_{\infty} \leq \varepsilon$, where $\|f\|_{\infty}=\max _{(x, t) \in[-1,1] \times[-1,1]}|f(x, t)|$.

The adaptation of this result to rectangular domains $[0, b] \times[0, a]$ is straightforward. In that case we also define

$$
\|f\|_{\infty}=\max _{(x, t) \in[0, b] \times[0, a]}|f(x, t)| .
$$

Let $J=[0, b] \times[0, a]$ and define $E(J)$ as the space of all continuous real-valued functions endowed with the norm

$$
\|f\|_{*}^{2}=\frac{1}{\pi^{2}} \int_{0}^{a} \int_{0}^{b}|f(x, t)|^{2} \frac{1}{\sqrt{a t-t^{2}}} \frac{1}{\sqrt{b x-x^{2}}} d x d t .
$$

In $[11,12,13]$ Chen et al. provides upper bounds for the truncation error of the two-variable Chebyshev series expansion (17). This will give us an idea of the accuracy of this approximation of the solution, in the cases where it presents some regularity. The first result does not demand high regularity assumptions on the solution, which is the expected situation in the fractional setting.

Theorem 2.3. Let $g$ be in $E(J)$, with $J=[0, b] \times[0, a]$, and such that, $\frac{\partial^{2} g}{\partial x \partial t} \in E(J)$. Let $P_{n, m}$ be defined by (17), then

$$
\left\|\left(I-P_{n, m}\right) g\right\|_{\infty} \leq B_{n m} Q
$$


where

$$
\begin{aligned}
B_{n m} & =\gamma(m)+\gamma(n)+\gamma(0)(\gamma(n)+\gamma(m)), \\
Q & =\max \left\{\left\|\frac{d h}{d t}\right\|_{*},\left\|\frac{d p}{d x}\right\|_{*},\left\|\frac{\partial^{2} g}{\partial x \partial t}\right\|_{*}\right\},
\end{aligned}
$$

and

$$
\begin{aligned}
\gamma^{2}(n) & =2 \sum_{j \geq n+1} \frac{1}{j^{2}}, \\
h(t) & =\frac{1}{\pi} \int_{0}^{b} g(x, t) \frac{1}{\sqrt{a t-t^{2}}} \frac{1}{\sqrt{b x-x^{2}}} d x, \\
p(x) & =\frac{1}{\pi} \int_{0}^{a} g(x, t) \frac{1}{\sqrt{a t-t^{2}}} \frac{1}{\sqrt{b x-x^{2}}} d t .
\end{aligned}
$$

The next Theorem requires more smoothness conditions to be satisfied.

Theorem 2.4. Let $g$ be in $E(J)$, with $J=[0, b] \times[0, a]$, and such that, $\frac{\partial^{k+l} g}{\partial x^{k} \partial t^{l}}$, $\frac{\partial^{s} g}{\partial x^{s}}, \frac{\partial^{s} g}{\partial t^{s}} \in E(J)$, for $1 \leq k \leq p, 1 \leq l \leq p, 0 \leq s \leq 2 p, p \geq 2$. Let $P_{n, m}$ be defined by (17), then

$$
\left\|\left(I-P_{n, m}\right) g\right\|_{\infty} \leq \tilde{B}_{n m} \tilde{Q},
$$

where

$$
\tilde{B}_{n m}=2\left(\frac{b a}{4}\right)^{2 p}\left(\frac{1}{\sqrt{n m} \prod_{k=0}^{p-2}(n-k) \prod_{k=0}^{p-2}(m-k)}+\frac{\sqrt{n+1}}{\sqrt{m} \prod_{k=0}^{2 p-2}(m-k)}+\frac{\sqrt{m+1}}{\sqrt{n} \prod_{k=0}^{2 p-2}(n-k)}\right)
$$

and

$$
\tilde{Q}=\max \left\{\left\|\frac{\partial^{2 p} g}{\partial x^{p} \partial t^{p}}\right\|_{*},\left\|\frac{\partial^{2 p} g}{\partial x^{2 p}}\right\|_{*},\left\|\frac{\partial^{2 p} g}{\partial t^{2 p}}\right\|_{*}\right\}
$$

Remark 2.1. From Theorems 2.3 and 2.4, it is clear that, as in the univariate case, the smoother the function $g(x, t)$ is, the faster the method converges. It is clear from these two Theorems that, if a certain function is smooth then

$$
\left\|\left(I-P_{n, m}\right) g\right\|_{\infty} \leq A_{n m} C,
$$

where $A_{n m}$ and $C$ are given by (19) and (20) or (21) and (22) depending on the smoothness of the function being approximated.

If we consider $m=n$ we easily see that $B_{n m} \sim \frac{C_{1}}{\sqrt{n}}$ while $\tilde{B}_{n m} \sim \frac{C_{2}}{n^{2 p-1}}$, with $C_{1}$ and $C_{2}$ constants. 


\section{The numerical scheme}

In this section we describe the numerical method. As we will see, the method presented in this work is faster than the typical finite difference approaches, where we need to compute all the diffusion history at each time step (cf. section of numerical results).

If the source function $f$, and the functions $\phi_{0}, \phi_{b}$ and $g_{0}$ related with the boundary and initial conditions, respectively, of the problem (7) satisfy the assumptions of Theorem 2.2 then the problem (7)-(9) has an unique solution $u(x, t) \in C_{t}([0, a]) \cap W_{t}^{1}((0, a)) \cap C_{x}^{2}((0, b))$. Furthermore if the solution $u$ is a function of bounded variation, as we remarked previously, based on a result from [62], it can be approximated by the truncated series $u_{n, m}(x, t)$ defined in (17).

First, to deal with the integral term in (7), we use a Gauss-Legendre quadrature formula with $s$ points, with associated weights and points denoted, respectively, by $\omega_{q}$ and $\beta_{q}$. Hence (7) is approximated with

$$
\frac{1}{2} \sum_{q=1}^{s} \omega_{q} c\left(\frac{\beta_{q}+1}{2}\right) \frac{\partial^{\frac{\beta_{q}+1}{2}} u_{n, m}(x, t)}{\partial t^{\frac{\beta_{q}+1}{2}}}=\frac{\partial^{2} u_{n, m}(x, t)}{\partial x^{2}}+f(x, t) .
$$

Using (11) the following expressions can be derived:

$$
\begin{aligned}
& \frac{\partial^{\alpha} u_{n, m}(x, t)}{\partial t^{\alpha}}=\sum_{i=\lceil\alpha\rceil}^{n} \sum_{j=0}^{m} a_{i j} T_{b, j}(x) \sum_{k=\lceil\alpha\rceil}^{i} w_{i, k}^{(\alpha)} t^{k-\alpha}, \\
& \frac{\partial^{2} u_{n, m}(x, t)}{\partial x^{2}}=\sum_{i=0}^{n} \sum_{j=2}^{m} a_{i j} T_{a, i}(t) \sum_{\gamma=2}^{j} \omega_{j, \gamma} x^{\gamma-2},
\end{aligned}
$$

where the weights $w_{i, k}^{(\alpha)}$ are defined by (see also [20])

$$
w_{i, k}^{(\alpha)}=(-1)^{i-k} \frac{2^{2 k} i(i+k-1) ! \Gamma(k+1)}{(i-k) !(2 k) ! \Gamma(k+1-\alpha) a^{k}},
$$

and the weights $\omega_{j, \gamma}$ are given by

$$
\omega_{j, \gamma}=(-1)^{j-\gamma} \frac{2^{2 \gamma} j(j+\gamma-1) ! \gamma(\gamma-1)}{(j-\gamma) !(2 \gamma) ! b^{\gamma}} .
$$

Inserting (24) and (25) in (23), we obtain the following approximation of equation (7):

$$
\begin{aligned}
& \frac{1}{2} \sum_{q=1}^{s} \omega_{q} c\left(\frac{\beta_{q}+1}{2}\right) \sum_{i=\left\lceil\frac{\beta_{q}+1}{2}\right\rceil}^{n} \sum_{j=0}^{m} a_{i j} T_{b, j}(x) \sum_{k=\left\lceil\frac{\beta_{q}+1}{2}\right\rceil}^{i} w_{i, k}^{\left(\frac{\beta_{q}+1}{2}\right)} t^{k-\frac{\beta_{q}+1}{2}}= \\
& \sum_{i=0}^{n} \sum_{j=2}^{m} a_{i j} T_{a, i}(t) \sum_{\gamma=2}^{j} \omega_{j, \gamma} x^{\gamma-2}+f(x, t) .
\end{aligned}
$$

In this case, since $\frac{\beta_{q}+1}{2} \in[0,1], q=1, \ldots, s$, then $\left\lceil\frac{\beta_{q}+1}{2}\right\rceil=1, q=1, \ldots, s$. Finally, in order to determine the coefficients $a_{i j}$ of the truncated Chebyshev series expansion, we will use collocation. 
For collocation points we consider, as usual, $t_{p}=\frac{a}{2}\left(\cos \left(\frac{\pi p}{n}\right)+1\right), p=0, \ldots, n$ and $x_{\ell}=$ $\frac{b}{2}\left(\cos \left(\frac{\pi \ell}{m}\right)+1\right), \ell=0, \ldots, m$.

The coefficients $a_{i j}, i=0, \ldots, n, \quad j=0, \ldots, m$, of the discrete solution are then computed by imposing that $u_{n, m}(x, t)$ satisfies the multi-term equation (28) at the collocation points $\left(x_{\ell}, t_{p}\right)$, $\ell=1, \ldots, m-1, p=0, \ldots, n-1$ :

$$
\begin{aligned}
& \frac{1}{2} \sum_{q=1}^{s} \omega_{q} c\left(\frac{\beta_{q}+1}{2}\right) \sum_{i=\left\lceil\frac{\beta_{q}+1}{2}\right\rceil}^{n} \sum_{j=0}^{m} a_{i j} T_{b, j}\left(x_{\ell}\right) \sum_{k=\left\lceil\frac{\beta_{q}+1}{2}\right\rceil}^{i} w_{i, k}^{\left(\frac{\beta_{q}+1}{2}\right)} t_{p}^{k-\frac{\beta_{q}+1}{2}}= \\
& \sum_{i=0}^{n} \sum_{j=2}^{m} a_{i j} T_{a, i}\left(t_{p}\right) \sum_{\gamma=2}^{j} \omega_{j, \gamma} x_{\ell}^{\gamma-2}+f\left(x_{\ell}, t_{p}\right) .
\end{aligned}
$$

On the other hand, we insist that the approximate solution, $u_{n, m}(x, t)$, satisfies the boundary and initial conditions at the collocation points placed at the boundary of the domain $[0, b] \times[0, a]$. Thus, using the fact that the shifted Chebyschev polynomials satisfy $T_{b, j}(0)=(-1)^{j}$ and $T_{b, j}(1)=1$, the coefficients $a_{i j}$ that define the approximate solution $u_{n, m}(x, t)$ of $u$ are such that it satisfies the system of equations (29) together with

$$
\begin{aligned}
& \sum_{i=0}^{n} \sum_{j=0}^{m} a_{i j} T_{a, i}\left(t_{p}\right)(-1)^{j}=\phi_{0}\left(t_{p}\right), \\
& \sum_{i=0}^{n} \sum_{j=0}^{m} a_{i j} T_{a, i}\left(t_{p}\right)=\phi_{b}\left(t_{p}\right), \\
& \sum_{i=0}^{n} \sum_{j=0}^{m} a_{i j}(-1)^{i} T_{b, j}\left(x_{\ell}\right)=g_{0}\left(x_{\ell}\right), \\
& p=0, \ldots, n-1, \ell=0, \ldots, m .
\end{aligned}
$$

This way, we have $(n+1) \times(m+1)$ unknown coefficients $a_{i j}$ and $(n+1) \times(m+1)$ equations.

\section{Error analysis}

Let $u(x, t)$ be the exact solution of problem $(7)-(9), u_{n, m}(x, t)$ the truncated series representation of $u$, given by (17), and denote by $\hat{a}_{i j}$ the solution of the linear system of equations (29)-(32). Let us define the approximate solution obtained with the method described in the previous section as

$$
\hat{u}_{n, m}(x, t)=\sum_{i=0}^{n} \sum_{j=0}^{m} \hat{a}_{i j} T_{a, i}(t) T_{b, j}(x), \quad(x, t) \in[0, b] \times[0, a] .
$$

In Theorem 2.4 an error bound for the norm of the difference between the exact solution, $u(x, t)$, and the approximate solution $u_{n, m}(x, t)$ corresponding to the truncated Chebyshev series has been provided. In the numerical method described in the previous section, we have also replaced the time-fractional and the space derivatives of $u$ by the time-fractional and the space derivatives of $u_{n, m}$. We begin this section with some auxiliary lemmas presenting error bound estimates for these approximations. 
We can then use the results in the previous section, namely Theorem 2.3, Theorem 2.4 and Remark 2.1, to obtain some upper bounds on the approximations considered here. From Remark 2.1, the following two Lemmas are straightforward to obtain.

Lemma 4.1. If $u(x, t)$ and at least the following partial derivative $\frac{\partial^{4} u}{\partial t \partial x^{3}}$ are continuous on $J$, then:

$$
\left\|\left(I-P_{n, m}\right) \frac{\partial^{2} u}{\partial x^{2}}\right\|_{\infty} \leq A_{n m} M_{x}
$$

where $A_{n m}$ and $M_{x}$ are defined according to Remark 2.1.

Lemma 4.2. If $u(x, t)$ and at least the following partial derivative $\frac{\partial^{3} u}{\partial t^{2} \partial x}$ are continuous on $J$, then:

$$
\left\|\left(I-P_{n, m}\right) \frac{\partial u}{\partial t}\right\|_{\infty} \leq A_{n m} M_{t},
$$

where $A_{n m}$ and $M_{t}$ are defined according to Remark 2.1.

Taking into account the definition of the Caputo derivative of order $\alpha$ of a function $u$ (see (5)), which for $0<\alpha<1$ reads

$$
\frac{\partial^{\alpha} u(t, x)}{\partial x^{\alpha}}=\frac{1}{\Gamma(1-\alpha)} \int_{0}^{t}(t-s)^{-\alpha} \frac{\partial u}{\partial s}(x, s) d s,
$$

and Lemma 4.2 we obtain the following result:

Lemma 4.3. If $u(x, t)$ and at least the following partial derivative $\frac{\partial^{3} u}{\partial t^{2} \partial x}$ are continuous on $J$, then:

$$
\left\|\left(I-P_{n, m}\right) \frac{\partial^{\alpha} u}{\partial t^{\alpha}}\right\|_{\infty} \leq M_{\alpha} A_{n m},
$$

with $M_{\alpha}=\frac{M_{t} a^{1-\alpha}}{\Gamma(1-\alpha)(1-\alpha)}$.

Proof.

$$
\begin{aligned}
\left\|\left(I-P_{n, m}\right) \frac{\partial^{\alpha} u}{\partial t^{\alpha}}\right\|_{\infty} & \leq \frac{1}{\Gamma(1-\alpha)} \int_{0}^{t}(t-s)^{-\alpha}\left\|\left(I-P_{n, m}\right) \frac{\partial u}{\partial s}\right\|_{\infty} d s \\
& \leq \frac{M_{t} A_{n, m}}{\Gamma(1-\alpha)} \int_{0}^{t}(t-s)^{-\alpha} d s \\
& \leq \frac{M_{t} A_{n m} t^{1-\alpha}}{(1-\alpha) \Gamma(1-\alpha)} \leq \frac{M_{t} A_{n m} a^{1-\alpha}}{(1-\alpha) \Gamma(1-\alpha)} .
\end{aligned}
$$

Before we present our main result in this section, we prove some auxiliary results. 
Proposition 4.1. The linear system of equations (29)-(32) can be equivalently written in the matrix form

$$
A z=B,
$$

where $A$ is invertible, $B$ is the vector with the corresponding error for each one of the equations given in system of equations (29)-(32), $z$ is the vector with components $\left(\hat{a}_{i j}-a_{i j}\right), i=0, \ldots, n, j=$ $0, \ldots, m$, and the following estimate holds

$$
\left\|\left[\hat{a}_{i j}-a_{i j}\right]\right\| \leq C_{1}\left(\|R(s, x, t)\|_{\infty}+\|E(n, m, x, t)\|_{\infty}\right),
$$

where $\|\cdot\|$ denotes the maximum vector or matrix norm, $R(s, x, t)$ is the error of the used s-point quadrature rule, and

$$
E(n, m, x, t)=\frac{1}{2} \sum_{q=1}^{s} \omega_{q} c\left(\frac{\beta_{q}+1}{2}\right)\left(I-P_{n, m}\right)\left(\frac{\partial^{\frac{\beta_{q}+1}{2}}}{\partial t^{\frac{\beta_{q}+1}{2}}} u(x, t)\right)+\left(I-P_{n, m}\right)\left(\frac{\partial^{2}}{\partial x^{2}} u(x, t)\right) .
$$

Proof. Let us define:

$$
\begin{aligned}
& \mathcal{L}_{1}(u(x, t))=\int_{0}^{1} c(\alpha) \frac{\partial^{\alpha}}{\partial t^{\alpha}} u(x, t) d \alpha-\frac{\partial^{2}}{\partial x^{2}} u(x, t), \\
& \mathcal{L}_{2}(u(x, t))=\frac{1}{2} \sum_{q=1}^{s} \omega_{q} c\left(\frac{\beta_{q}+1}{2}\right) \frac{\partial^{\frac{\beta q+1}{2}}}{\partial t^{\frac{\beta q+1}{2}}} u(x, t)-\frac{\partial^{2}}{\partial x^{2}} u(x, t) .
\end{aligned}
$$

Note that the collocation equations (29) can be written as

$$
\begin{aligned}
& \mathcal{L}_{2}\left(\hat{u}_{n, m}\left(x_{\ell}, t_{p}\right)\right)=f\left(x_{\ell}, t_{p}\right)=\mathcal{L}_{1}\left(u\left(x_{\ell}, t_{p}\right)\right) \\
& \Leftrightarrow \mathcal{L}_{2}\left(\hat{u}_{n, m}\left(x_{\ell}, t_{p}\right)\right)=\mathcal{L}_{1}\left(u\left(x_{\ell}, t_{p}\right)\right)-\mathcal{L}_{2}\left(u\left(x_{\ell}, t_{p}\right)\right)+\mathcal{L}_{2}\left(u\left(x_{\ell}, t_{p}\right)\right)-\mathcal{L}_{2}\left(u_{n, m}\left(x_{\ell}, t_{p}\right)\right)+\mathcal{L}_{2}\left(u_{n, m}\left(x_{\ell}, t_{p}\right)\right) \\
& \ell=1, \ldots, m-1, \quad p=0, \ldots, n-1 .
\end{aligned}
$$

and then

$$
\mathcal{L}_{2}\left(\left(\hat{u}_{n, m}-u_{n, m}\right)\left(x_{\ell}, t_{p}\right)\right)=\left[\mathcal{L}_{1}\left(u\left(x_{\ell}, t_{p}\right)\right)-\mathcal{L}_{2}\left(u\left(x_{\ell}, t_{p}\right)\right)\right]+\left[\mathcal{L}_{2}\left(u\left(x_{\ell}, t_{p}\right)\right)-\mathcal{L}_{2}\left(u_{n, m}\left(x_{\ell}, t_{p}\right)\right)\right]
$$

It is obvious that $\mathcal{L}_{1}(u(x, t))-\mathcal{L}_{2}(u(x, t))$ corresponds to the error of the used $s$-points quadrature rule, say $R(s, x, t)$, and then (see [5]):

$$
\begin{aligned}
{\left[\mathcal{L}_{1}\left(u\left(x_{\ell}, t_{p}\right)\right)-\mathcal{L}_{2}\left(u\left(x_{\ell}, t_{p}\right)\right)\right] } & =R\left(s, x_{\ell}, t_{p}\right)=\frac{((s) !)^{4}}{(2 s+1)((2 s) !)^{4}} \frac{\partial^{2 s} G}{\partial \alpha^{2 s}}\left(\theta, x \ell, t_{p}\right) \\
& \approx \frac{\pi}{4^{s}} \frac{\partial^{2 s} G}{\partial \alpha^{2 s}}\left(\theta, x_{\ell}, t_{p}\right), \quad \theta \in[0,1]
\end{aligned}
$$

where the function $G$ is defined by

$$
G(\alpha, x, t)=c\left(\frac{\alpha+1}{2}\right) \frac{\partial^{\frac{\alpha+1}{2}}}{\partial t^{\frac{\alpha+1}{2}}} u(x, t) .
$$


On the other hand,

$$
\left[\mathcal{L}_{2}\left(u\left(x_{\ell}, t_{p}\right)\right)-\mathcal{L}_{2}\left(u_{n, m}\left(x_{\ell}, t_{p}\right)\right)\right]=E\left(n, m, x_{\ell}, t_{p}\right) .
$$

Substituting (40) and (42) in (39), we conclude that the collocation equations (29) can be written as:

$$
\mathcal{L}_{2}\left(\left(\hat{u}_{n, m}-u_{n, m}\right)\left(x_{\ell}, t_{p}\right)\right)=R\left(s, x_{\ell}, t_{p}\right)+E\left(n, m, x_{\ell}, t_{p}\right) .
$$

Taking into account the boundary and initial conditions ((8) and (9)) and equations (30)-(32) we have that:

$$
\begin{aligned}
\sum_{i=0}^{n} \sum_{j=0}^{m}\left(a_{i j}-\hat{a}_{i j}\right)(-1)^{j} T_{i}\left(t_{p}\right)= & \left(\hat{u}_{n, m}-u_{n, m}\right)\left(0, t_{p}\right)=\left(u-u_{n, m}\right)\left(0, t_{p}\right) \\
= & \left(I-P_{n, m}\right)\left(u\left(0, t_{p}\right)\right)=E\left(n, m, 0, t_{p}\right) \\
\sum_{i=0}^{n} \sum_{j=0}^{m}\left(a_{i j}-\hat{a}_{i j}\right) T_{i}\left(t_{p}\right)= & \left(\hat{u}_{n, m}-u_{n, m}\right)\left(b, t_{p}\right)=\left(u-u_{n, m}\right)\left(b, t_{p}\right) \\
= & \left(I-P_{n, m}\right)\left(u\left(b, t_{p}\right)\right)=E\left(n, m, b, t_{p}\right), \\
\sum_{i=0}^{n} \sum_{j=0}^{m}\left(a_{i j}-\hat{a}_{i j}\right)(-1)^{i} T_{j}\left(x_{\ell}\right)= & \left(\hat{u}_{n, m}-u_{n, m}\right)\left(x_{\ell}, 0\right)=\left(u-u_{n, m}\right)\left(x_{\ell}, 0\right) \\
= & \left(I-P_{n, m}\right)\left(u\left(x_{\ell}, 0\right)\right)=E_{\left(x_{\ell}, 0\right)}\left(n, m, x_{\ell}, 0\right), \\
& p=0, \ldots, n-1, \ell=0, \ldots, m .
\end{aligned}
$$

Then, from (39) and (44)-(46) it follows that equations (29)-(32) may be rewritten in the form

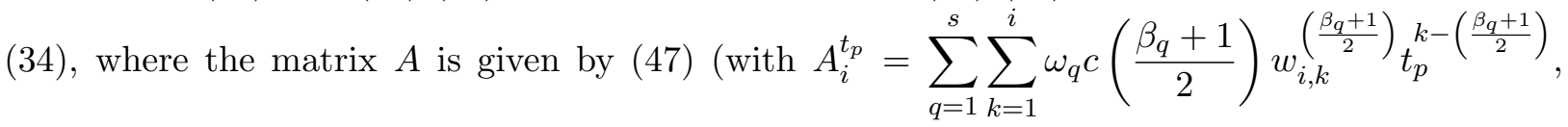
and $\left.B_{m}^{x_{l}}=\sum_{k=2}^{m} \omega_{m, k} x_{l}^{m-2}\right)$ and vector $B$ is given by (48) (with $R_{s}^{x_{\ell}, t_{p}}=R\left(s, x_{\ell}, t_{p}\right)$ and $E_{n, m}^{x_{\ell}, t_{p}}=$ $\left.E\left(n, m, x_{\ell}, t_{p}\right)\right)$.

It can be checked that $\mathrm{A}$ is an invertible matrix if the collocation points are all distinct. We 
have verified it computationally for several values of $n$ and $m$.

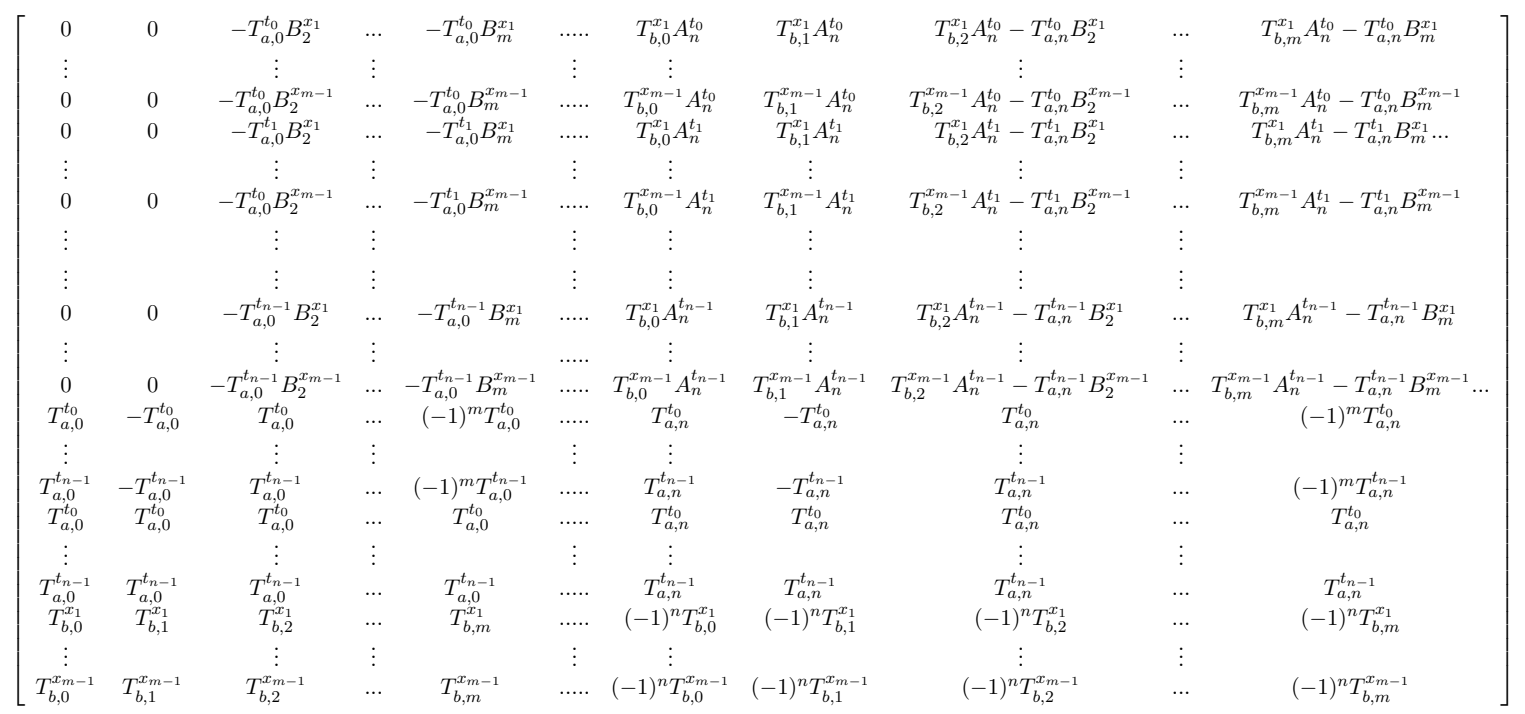

$$
\begin{aligned}
& {\left[R_{s}^{x_{1}, t_{0}}+E_{n, m}^{x_{1}, t_{0}}, \ldots, R_{s}^{x_{m-1}, t_{0}}+E_{n, m}^{x_{m-1}, t_{n-1}}, E_{n, m}^{0, t_{0}}, \ldots, E_{n, m}^{0, t_{n-1}}, E_{n, m}^{b, t_{0}}, \ldots, E_{n, m}^{b, t_{n-1}},, E_{n, m}^{x_{0}, 0}, \ldots, E_{n, m}^{x_{m}, 0}\right]^{T}}
\end{aligned}
$$

Being $A$ an invertible matrix, then

$$
\begin{aligned}
\|z\|=\left\|\left[\hat{a}_{i j}-a_{i j}\right]\right\| & \leq\left\|A^{-1}\right\|\left(\left\|R\left(s, x_{\ell}, t_{p}\right)\right\|+\left\|E\left(n, m, x_{\ell}, t_{p}\right)\right\|\right) \\
& \leq C_{1}\left(\|R(s, x, t)\|_{\infty}+\|E(n, m, x, t)\|_{\infty}\right) .
\end{aligned}
$$

Proposition 4.2. Let $u$ be the solution of the problem (7)-(9). If

- $G(\alpha, x, t) \in C^{2 s}([0,1])$, for fixed $(x, t) \in[0, b] \times[0, a]$, with $G$ defined by (41),

- $u(x, t), \frac{\partial^{3} u}{\partial t^{2} \partial x}$ and $\frac{\partial^{4} u}{\partial t \partial x^{3}}$ are continuous on $J$,

then,

$$
\left\|u_{n, m}-\hat{u}_{n, m}\right\|_{\infty} \leq C_{4}\left(4^{-s}+A_{n m}\right),
$$

where $A_{n m}$ is defined according to Remark 2.1.

Proof. Note that since $\left\|T_{a, i}\right\| \leq 1$ and $\left\|T_{b, j}\right\| \leq 1$ we have

$$
\begin{aligned}
\left\|\hat{u}_{n, m}-u_{n, m}\right\|_{\infty} & \leq \sum_{i=0}^{n} \sum_{j=0}^{m}\left|\hat{a}_{i, j}-a_{i, j}\right|\left\|T_{a, i}\right\|_{\infty}\left\|T_{b, j}\right\|_{\infty} \leq\left\|\left[\hat{a}_{i, j}-a_{i, j}\right]\right\|_{1} \leq C^{*}\left\|\left[\hat{a}_{i, j}-a_{i, j}\right]\right\|_{\infty} \\
& \leq C^{*} C_{1}\left(\|R(s, x, t)\|_{\infty}+\|E(n, m, x, t)\|_{\infty}\right) .
\end{aligned}
$$

Under the conditions on the function $G$, from (40) it follows

$$
\max _{(x, t) \in[0, b] \times[0, a]}|R(s, x, t)|=\|R(s, x, t)\|_{\infty} \leq \frac{C_{2}}{4^{s}} .
$$


On the other hand, taking into account Lemmas 4.1 and 4.3 we easily come to the conclusion that

$$
\|E(n, m, x, t)\|_{\infty} \leq C_{3} A_{n m} .
$$

Hence, the result is proved with $C_{4}=\max \left\{C_{2}, C_{3}\right\}$.

Defining the error function,

$$
e_{n, m}(x, t)=u(x, t)-\hat{u}_{n, m}(x, t)
$$

we finally present our main result of this section.

Theorem 4.1. Assume that all the conditions of Proposition 4.2 are satisfied. Then

$$
\left\|e_{n, m}\right\|_{\infty} \leq C\left(4^{-s}+A_{n m}\right) .
$$

Proof. First note that:

$$
\left\|e_{n, m}\right\|_{\infty} \leq\left\|u-u_{n, m}\right\|_{\infty}+\left\|u_{n, m}-\hat{u}_{n, m}\right\|_{\infty} .
$$

Under the conditions stated in Theorem 2.3 or Theorem 2.4 in the case of a smoother solution (see also Remark 2.1), the following upper bound for $\left\|u-u_{n, m}\right\|_{\infty}$ can be derived:

$$
\left\|u-u_{n, m}\right\|_{\infty} \leq M A_{n m} C .
$$

Taking this and the result in Proposition 4.2 into account, the theorem is proved.

\section{Numerical results}

In order to analyse the accuracy of the proposed numerical method, we consider the $L_{\infty}$ errors:

$$
\left\|\mathbf{E}_{n, m}\right\|_{\infty}=\max _{i, j}\left|u\left(x_{i}, t_{j}\right)-u_{n, m}\left(x_{i}, t_{j}\right)\right|, \text { for }\left(x_{i}, t_{j}\right) \in[0, b] \times[0, a] .
$$

In order to approximate the integral that defines the distributed order derivative we use a 3 -point Gaussian quadrature formula.

To illustrate the theoretical results, we first consider two examples in which the solution satisfies the required smoothness assumptions in the convergence analysis. Because we can expect the solution of fractional differential equations to be nonsmooth we also consider some examples with nonsmooth solutions to check the performance of the present numerical method in such cases.

\subsection{Numerical results obtained with the proposed method}

In this subsection we consider some examples in order to illustrate the performance and feasibility of the proposed method. First we present the $L_{\infty}$ error obtained for the examples shown below, and then we study in more detail the examples whose solutions do not satisfy the regularity assumptions required for the theoretical convergence analysis. Even for these cases the numerical method showed reasonable results. 


\section{Example 5.1.}

$$
c(\alpha)=\Gamma(4-\alpha) ; \quad f(x, t)=t^{2}\left(\frac{x(6 t+t \log (t)-6) \sin (x)}{\log (t)}-2 t \cos (x)\right),
$$

with analytical solution given by $u(x, t)=t^{3} x \sin (x), \quad(x, t) \in[0,1] \times[0,1]$.

\section{Example 5.2.}

$$
c(\alpha)=\Gamma\left(\frac{7}{2}-\alpha\right) ; \quad f(x, t)=\frac{t^{3 / 2}\left(15 \sqrt{\pi}(t-1)(x-1)^{2} x+16 t(2-3 x) \log (t)\right)}{8 \log (t)},
$$

with analytical solution given by $u(x, t)=t^{5 / 2} x(1-x)^{2}, \quad(x, t) \in[0,1] \times[0,1]$.

\section{Example 5.3.}

$$
c(\alpha)=\Gamma\left(\frac{5}{2}-\alpha\right) ; \quad f(x, t)=\frac{\sqrt{t}(x-1)^{2}\left(3 \sqrt{\pi}(t-1)(x-1)^{2} x^{2}-8 t(5 x(3 x-2)+1) \log (t)\right)}{4 \log (t)},
$$

with analytical solution given by $u(x, t)=t^{3 / 2} x^{2}(1-x)^{4}, \quad(x, t) \in[0,1] \times[0,1]$. Note that in this case the solution does not satisfy the smoothness conditions required in the convergence analysis of the method described in the previous sections. Even so, we tested the method in this example and in the next section we compare the obtained numerical results with the ones obtained with the finite difference scheme in [25] (Note that this example does not satisfy also the regularity assumptions demanded in the convergence analysis in [25]).

\begin{tabular}{c||ccc}
\hline \multicolumn{1}{c||}{} & \multicolumn{3}{|c}{$\left\|\mathbf{E}_{n, m}\right\|_{\infty}$} \\
\cline { 2 - 4 }$n=m$ & Example 5.1 & Example 5.2 & Example 5.3 \\
\hline \multicolumn{1}{c||}{} & & & \\
1 & $3.142 \cdot 10^{-1}$ & $1.481 \cdot 10^{-2}$ & $2.193 \cdot 10^{-2}$ \\
3 & $3.469 \cdot 10^{-3}$ & $3.313 \cdot 10^{-4}$ & $1.297 \cdot 10^{-2}$ \\
5 & $8.382 \cdot 10^{-6}$ & $2.397 \cdot 10^{-5}$ & $1.207 \cdot 10^{-3}$ \\
7 & $1.275 \cdot 10^{-8}$ & $4.761 \cdot 10^{-6}$ & $1.059 \cdot 10^{-5}$ \\
9 & $1.020 \cdot 10^{-11}$ & $1.429 \cdot 10^{-6}$ & $4.473 \cdot 10^{-6}$ \\
11 & $3.709 \cdot 10^{-14}$ & $4.402 \cdot 10^{-7}$ & $1.685 \cdot 10^{-6}$ \\
\hline
\end{tabular}

Table 1: The errors $\left\|\mathbf{E}_{n, m}\right\|_{\infty}$, for several values of $n$ and $m$, from Examples 5.1, 5.2 and 5.3.

In Table 1 we list the $L_{\infty}$ errors obtained with the proposed method, for examples 5.1, 5.2 and 5.3 , with several values of $n$ and $m$. It can be observed that the error is smaller for the biggest values of $m$ and $n$, that we consider. Thus, the overall errors can be made smaller by adding new terms from the series (17) that approximate $u(x, t)$. This leads us to the conclusion that the convergence of the method can be observed even if the solution does not satisfy the regularity assumptions required for the convergence analysis.

However we observe more accuracy and faster convergence for the examples whose solutions are more regular. For example 5.1 the solution is $C^{\infty}([0,1])$, and therefore, the rules dictated by 
the convergence analysis presented before can be applied and we observe that is possible reduce the absolute error to very small values $\left(\sim 10^{-14}\right)$ with a reasonable number of terms in the double Chebyshev series.

In Figure 1 we present the absolute error for the Chebyschev series $\left|u_{10,10}(x, t)\right|$ obtained for the examples 5.1, 5.2 and 5.3. As we expected the absolute error is smaller for the solution that presents more regularity.
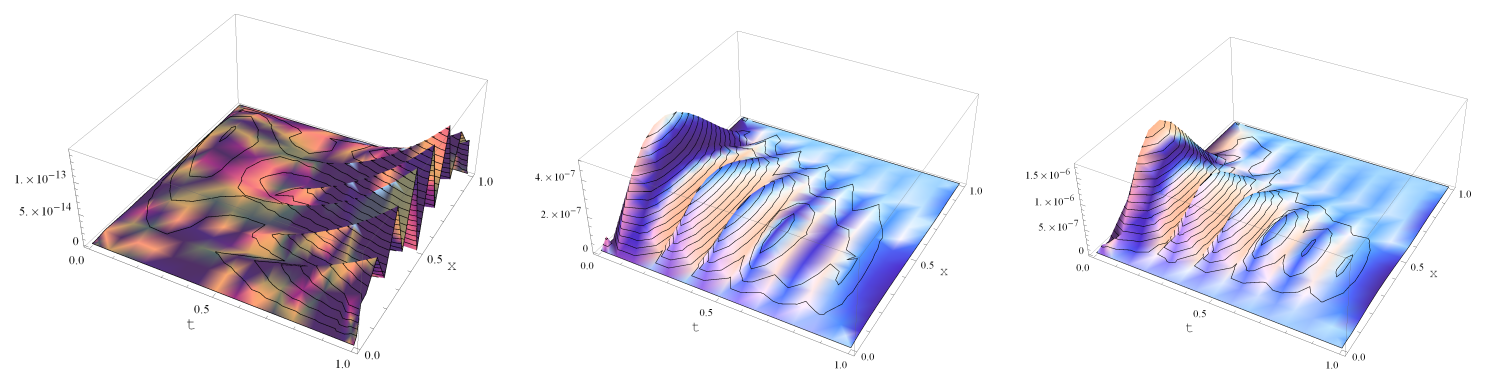

Figure 1: Plot of error function $\left|u(x, t)-u_{10,10}(x, t)\right|$. Left: Example 5.1. Center: Example 5.2. Right: Example 5.3.

Let us consider a final set of examples whose first derivative with respect to time is not defined at $t=0$. Since estimates for the error, when the solution is non-smooth at $t=0$, are not available in the literature, we have performed a large set of computations considering solutions with an increasing degree of singularity $(d)$.

\section{Example 5.4.}

$$
c(\alpha)=\Gamma\left(\frac{d+1}{d}-\alpha\right) ; \quad f(x, t)=\frac{(x-1)^{2}\left(\frac{(t-1)(x-1)^{2} x^{2} \Gamma\left(\frac{d+1}{d}\right)}{\log (t)}-2 t(5 x(3 x-2)+1)\right)}{t^{d / d+1}},
$$

with analytical solution given by $u(x, t)=t^{1 / d}(1-x)^{4} x^{2} ;, \quad(x, t) \in[0,1] \times[0,1]$. The following set of values for $d$ were considered $d=\{1,2,3, \ldots, 9,10,12,14,16, \ldots, 28,30\}$.

The error $\left\|\boldsymbol{E}_{n, m}\right\|_{\infty}$ obtained is shown in Figure 2. It can be seen that the error decreases asymptotically with the increase of $1 / d$, and that a slow convergence is obtained with the increase of $m=n$. Although slow, convergence can be observed, even for the most critical case.

\subsection{Comparison with an implicit finite difference scheme}

The method presented in this work was compared with the implicit numerical method proposed for these problems in [25]. For the numerical method proposed in [25] we consider a finite difference scheme of order $2-\alpha$ to approximate each fractional derivative, the midpoint rule (with stepsize $h$ ) to approximate the integral and a finite difference scheme of order 2 to approximate the second derivative in space. All the numerical experiments have been coded in MATHEMATICA and run on a personal computer with processor Intel(R) Core(TM) i5, $2.60 \mathrm{GHz}$ under operating system MICROSOFT WINDOWS 8.1. 


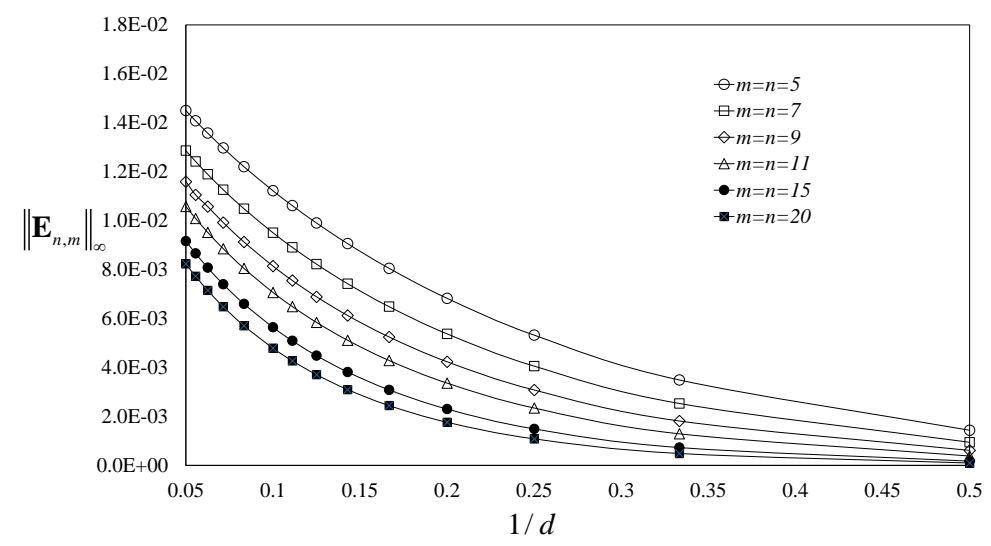

Figure 2: Variation of the error $\left\|\boldsymbol{E}_{n, m}\right\|_{\infty}$ with an increasing degree of the singularity.

In Tables 2 and 3 we list the maximum of the absolute errors in the points of the considered time and space mesh, that is, points $\left(x_{i}, t_{j}\right)$, where $x_{i}=i \Delta x, t_{j}=j \Delta t$, being $\Delta x$ and $\Delta t$ the space and time stepsizes respectively.

We also compare these results with the ones obtained with the proposed method (considering several values of $n$ and $m$ ), by computing the maximum of the absolute errors in the same mesh points where the absolute errors for the finite difference scheme were computed. The computational cost of each method is also presented.

From the results listed in Tables 2 and 3, we observe that with the method proposed in this work we can obtain an approximate solution with more accuracy and, by looking at the relative CPU times, with a lower computational cost.

The experimental convergence order of the method can be seen in figure 3. As expected, the convergence slows for the less regular solutions.

\begin{tabular}{|c|c|c|c|c|c|c|}
\hline \multirow[b]{2}{*}{$\Delta t$} & \multirow[b]{2}{*}{$h=\Delta x$} & \multirow{2}{*}{\multicolumn{2}{|c|}{ Implicite finite diff. scheme }} & \multicolumn{3}{|c|}{ Chebyshev Expansion } \\
\hline & & & & $m=n=5$ & $m=n=7$ & $m=n=9$ \\
\hline & & $\mid E \|$ & $\mathrm{CPU}$ & $\|E\|$ & $\|E\|$ & $\|E\|$ \\
\hline 0.25 & 0.5 & $5.06 \cdot 10^{-3}$ & 0.010 & $8.402 \cdot 10^{-6}$ & $1.275 \cdot 10^{-8}$ & $9.431 \cdot 10^{-12}$ \\
\hline 0.0625 & 0.25 & $1.60 \cdot 10^{-3}$ & 0.875 & $7.440 \cdot 10^{-6}$ & $1.275 \cdot 10^{-8}$ & $9.431 \cdot 10^{-12}$ \\
\hline 0.015625 & 0.125 & $2.80 \cdot 10^{-4}$ & 74.766 & $7.440 \cdot 10^{-6}$ & $1.275 \cdot 10^{-8}$ & $9.431 \cdot 10^{-12}$ \\
\hline 0.00390625 & 0.0625 & $4.97 \cdot 10^{-5}$ & 4938.58 & $8.225 \cdot 10^{-6}$ & $1.275 \cdot 10^{-8}$ & $9.7358 \cdot 10^{-12}$ \\
\hline & & & & CPU: 6.828 & CPU: 37.281 & CPU: 125.031 \\
\hline
\end{tabular}

Table 2: Maximum of errors $(\|E\|)$ and running-time (CPU) in seconds, obtained for Example 5.1.

\section{Conclusions}

In this work we have developed a new numerical method for the solution of distributed order time-fractional diffusion equations, based on the approximation of the solution by a Chebyshev truncated double series, and the subsequent collocation of the resulting discretised system of equations at suitable collocation points. We present a brief but clear introduction to the connection 


\begin{tabular}{|c|c|c|c|c|c|c|}
\hline \multirow[b]{2}{*}{$\Delta t$} & \multirow[b]{2}{*}{$h=\Delta x$} & \multirow{2}{*}{\multicolumn{2}{|c|}{ Implicite finite diff. scheme }} & \multicolumn{3}{|c|}{ Chebyshev Expansion } \\
\hline & & & & $m=n=5$ & $m=n=7$ & $m=n=9$ \\
\hline & & $\mid E \|$ & $\mathrm{CPU}$ & $\|E\|$ & $\|E\|$ & $\|E\|$ \\
\hline 0.25 & 0.5 & $8.40 \cdot 10^{-3}$ & 0.016 & $1.007 \cdot 10^{-3}$ & $2.054 \cdot 10^{-6}$ & $6.001 \cdot 10^{-7}$ \\
\hline 0.0625 & 0.25 & $2.45 \cdot 10^{-3}$ & 0.734 & $1.074 \cdot 10^{-3}$ & $5.705 \cdot 10^{-6}$ & $2.199 \cdot 10^{-6}$ \\
\hline 0.015625 & 0.125 & $6.36 \cdot 10^{-4}$ & 70.75 & $1.074 \cdot 10^{-3}$ & $1.048 \cdot 10^{-5}$ & $5.071 \cdot 10^{-6}$ \\
\hline 0.00390625 & 0.0625 & $1.62 \cdot 10^{-4}$ & 5347 & $1.185 \cdot 10^{-3}$ & $1.05 \cdot 10^{-5}$ & $5.338 \cdot 10^{-6}$ \\
\hline & & & & CPU: 4.391 & CPU: 21.531 & CPU: 72.766 \\
\hline
\end{tabular}

Table 3: Maximum of errors $(\|E\|)$ and running-time (CPU) in seconds, obtained for Example 5.3.

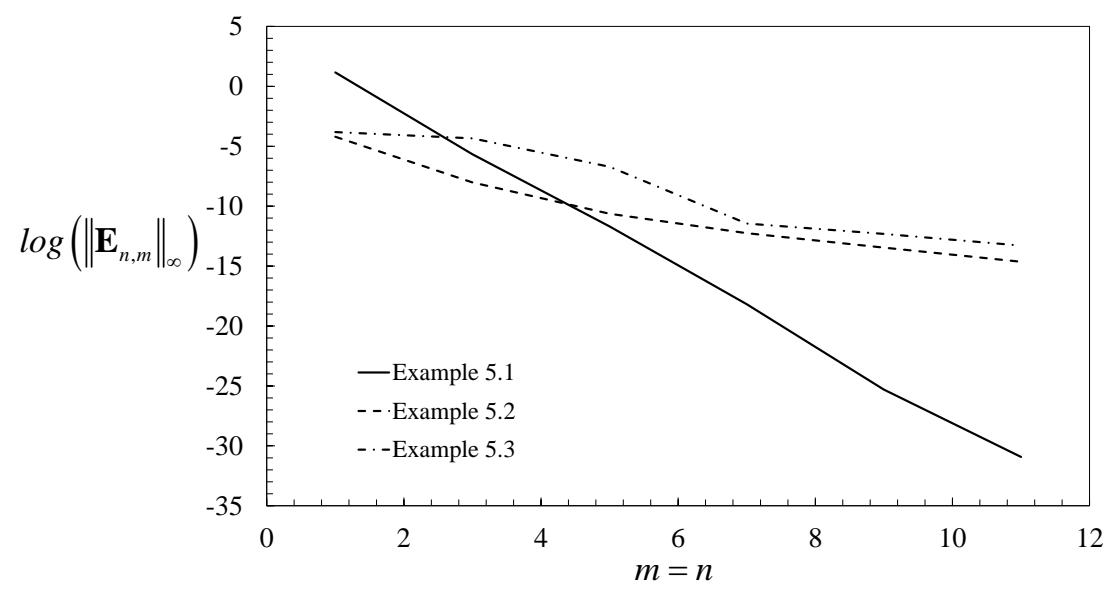

Figure 3: Variation of the logarithm of the error $\left\|\boldsymbol{E}_{n, m}\right\|_{\infty}$ with $m=n$, for Examples 5.1, 5.2 and 5.3

between random walks and fractional differential calculus, we review the existing papers on the subject of numerical solution for this type of equations, and, we also present a detailed error analysis for the proposed numerical method.

\section{Acknowledgements}

The work of the first author was financed by Portuguese Funds through FCT - Fundação para a Ciência e a Tecnologia, within the Project UID/MAT/00013/2013. The work of the second author was partially supported by the Fundação para a Ciência e a Tecnologia (Portuguese Foundation for Science and Technology) through the project UID/MAT/00297/2013 (Centro de Matemática e Aplicações). Luis L. Ferrás would like to thank the financial funding by FEDER through the COMPETE 2020 Programme, the National Funds through FCT - Portuguese Foundation for Science and Technology under the project UID/CTM/50025/2013, and also the financial funding by FCT through the scholarship SFRH/BPD/100353/2014.

\section{References}

[1] L. Bachelier, The Theory of Speculation, Ph.D. Thesis, 1900. 
[2] R. L. Bagley and P. J. Torvik, On the existence of the order domain and the solution of distributed order equations, International Journal of Applied Mathematics vol. I (2000) 865-882.

[3] R. L. Bagley and P. J. Torvik, On the existence of the order domain and the solution of distributed order equations, International Journal of Applied Mathematics vol. II (2000) 965-987.

[4] J.-P. Bouchaud, A. Georges, Anomalous diffusion in disordered media: Statistical mechanisms, models and physical applications, Phys. Rep. 195 (1990) 127-293.

[5] R. Burden and J. Faires. (2011). Numerical Analysis. Brooks-Cole Publishing Company, 9th Edition.

[6] M. Caputo, Elasticità e Dissipazione, Zanichelli, Bologna, Italy, 1969.

[7] M. Caputo, Mean fractional-order-derivatives differential equations and filters, Annali dellUniversità di Ferrara. Nuova Serie. Sezione VII. Scienze Matematiche 41 (1995) 73-84.

[8] M. Caputo, Distributed order differential equations modelling dielectric induction and diffusion, Fract. Calc. Appl. Anal. 4 (2001) 421-442.

[9] A.V. Chechkin, R. Gorenflo, and I. M. Sokolov, Retarding subdiffusion and accelerating superdiffusion governed by distributed-order fractional diffusion equations, Phys. Rev. E 66 (2002) 046129.

[10] A.V. Chechkin, J. Klafter, I.M. Sokolov, Fractional Fokker-Planck equation for ultraslow kinetics, Europhys. Lett. 63 (2003) 326-332.

[11] B. Chen, R. Garcia, L. Jódar, Frobenius-Chebyshev polynomial approximations with a priori error bounds for nonlinear intial value differential problems, Comput. Math. Appl. 41 (2001) 269-280.

[12] B. Chen, R. Garcia-Bolós, L. Jódar and M. D. Roselló, The Truncation Error of the Two-Variable Chebyshev Series Expansions, Comput. Math. Appl. 45 (2003) 1647-1653.

[13] B. Chen, R. Garcia-Bolós, L. Jódar and M. D. Roselló, Chebyshev polynomial approximations for nonlinear differential initial value problems, Nonlinear Analysis 63 (2005) e629-e637.

[14] H. Chen, S. Lu, W. Chen, Finite difference/spectral approximations for the distributed order time fractional reaction-diffusion equation on an unbounded domain, Journal of Computational Physics 315 (2016) 84-97.

[15] A. Compte, Stochastic foundations of fractional dynamics, Phys. Rev. E 53 (1996) 4191.

[16] K. Diethelm, N.J. Ford, Numerical solution methods for distributed order differential equations, Fract. Calc. Appl. Anal. 4 (2001) 531-542.

[17] K. Diethelm, N.J. Ford, A.D. Freed, Yu Luchko, Algorithms for the fractional calculus: a selection of numerical methods, Comput. Methods Appl. Mech. Eng. 194 (2005) 743-773.

[18] K. Diethelm, N.J. Ford, Numerical analysis for distributed-order differential equations, J. Comput. Appl. Math. 225 (2009) 96-104.

[19] K. Diethelm, The analysis of fractional differential equations: An application-oriented exposition using differential operators of Caputo type, Springer, Heidelberg, New York, 2010.

[20] E.H. Doha, A.H. Bhrawy and S.S. Ezz-Eldien, A Chebyshev spectral method based on operational matrix for initial and boundary value problems of fractional order, Comput. Math. Appl. 62 (2011) 2364-2373.

[21] P.L.T. Duong, , Ezra Kwok, and Moonyong Lee, Optimal design of stochastic distributed order linear SISO systems using hybrid spectral method, Math Probl. Eng. 2015 (2015).

[22] A. Einstein, in: R. Fürth (Ed.), Investigations on the theory of the Brownian movement, Dover, New York, 1956.

[23] H.C. Fogedby, Lévy flights in random environments, Phys. Rev. Lett. 73 (1994) 2517.

[24] N.J. Ford, M.L. Morgado, Distributed order equations as boundary value problems, Comput. Math. Appl. 64 (2012) 2973-2981.

[25] N.J. Ford, M.L. Morgado, M. Rebelo, An implicit finite difference approximation for the solution of the diffusion equation with distributed order in time, Electron. Trans. Numer. Anal. 44 (2015) 289-305.

[26] D. Froemberg, E. Barkai, Random time averaged diffusivities for Lévy walks, Euro. Phys. J. B 86 (2013) 1-13.

[27] G.-h. Gao, H.-w. Sun, Z.-z. Sun, Some high-order difference schemes for the distributed-order differential equations, J. Computat. Phys. 298 (2015) 337-359.

[28] G.-h. Gao, Z.-z. Sun, Two unconditionally stable and convergent difference schemes with the extrapolation method for the one-dimensional distributed-order differential equations, Numer. Meth. Part. D. E. 32 (2016) $591-615$.

[29] G.-h. Gao, Z.-z. Sun, Two Alternating Direction Implicit Difference Schemes for Two-Dimensional DistributedOrder Fractional Diffusion Equations, Comput. Math. Appl., 69 (2015) 926-948.

[30] R. Gorenflo, Y. Luchko, M. Stojanovic, Fundamental solution of a distributed order time-fractional diffusionwave equation as probability density, Fract. Calc. \& Appl. Anal. 16 (2013) 297-316.

[31] R. Gorenflo, F. Mainardi, E. Scalas, M. Raberto, in: K. Michael, T. Shanjian (Eds.), Fractional calculus and continuous-time finance III: the diffusion limit, Mathematical Finance. Birkhauser, Basel, 2001, pp. 171-180. 
[32] F. Höfling and T. Franosch, Anomalous transport in the crowded world of biological cells, Rep. Progr. Phys. 76 (2013) 046602.

[33] X. Hu, F. Liu, I. Turner, V. Anh, An implicit numerical method of a new time distributed-order and two-sided space-fractional advection-dispersion equation, Numer. Algorithms (2015) 1-15.

[34] S. Jespersen, R. Metzler, H. C. Fogedby, Lévy flights in external force fields: Langevin and fractional FokkerPlanck equations and their solutions, Phys. Rev. E 59 (1999) 2736.

[35] B. Jin, R. Lazarov, D. Sheen, Z. Zhou, Error estimates for approximations of distributed order time fractional diffusion with nonsmooth data, Frac. Calc. Appl. Anal. 19 (2016) 69-93.

[36] M.M. Khader, N.H. Swelam and A.M.S. Mahdy, Two computational algorithms for the numerical solution for system of fractional differential equations, Arab. J. Math. Sci. 21 (2015) 39-52.

[37] E. Kappler, Versuche zur Messung der Avogardo-Loschmidtschen Zahl aus der Brownschen Bewegung einer Drehwaage, Ann. Phys. 11 (1931) 233.

[38] J.T. Katsikadelis, Numerical solution of distributed order fractional differential equations, J. Comput. Phys. 259 (2014) 11-22.

[39] J. Klafter, M.F. Shlesinger, G. Zumofen, Beyond Brownian Motion, Phys. Today 49 (1996) 33-39.

[40] A. N. Kochubei, Distributed order calculus and equations of ultraslow diffusion, J. Math. Anal. Appl. 340 (2008) $252-281$.

[41] X.Y. Li, B. Y. Wu, A numerical method for solving distributed order diffusion equations, Appl. Math. Lett. 53 (2016) 92-99.

[42] Z. Li, Y. Luchko, and M. Yamamoto. Asymptotic estimates of solutions to initial-boundary-value problems for distributed order time-fractional diffusion equations, Frac. Calc. Appl. Anal. 17 (2014) 1114-1136.

[43] H.L. Liao, Y.N. Zhang, Y. Zhao, H.S. Shi, Stability and Convergence of Modified Du Fort-Frankel Schemes for Solving Time-Fractional Subdiffusion Equations, J. Sci. Comput. 61 (2014) 629-648.

[44] Y. Luchko. Boundary value problems for the generalised time fractional diffusion equation of distributed order, Frac. Cal. Appl. Anal. 12 (2009) 409-422.

[45] F. Mainardi, G. Pagnini, A. Mura, R. Gorenflo, Time-fractional diffusion of distributed order, J. Vib. Control 14 (2008) 1267-1290.

[46] F. Mainardi, M. Raberto, R. Gorenflo, E. Scalas, Fractional calculus and continuous-time finance II: the waitingtime distribution, Physica A 287 (2000) 468-481.

[47] B.B. Mandelbrot, The fractal geometry of nature, W.H. Freeman and Company, New York, 1982.

[48] J.C. Mason, Chebyshev Polynomial Approximations for the $L$ Membrane Eigenvalue Problem, SIAM J. Appl. Math. 15 (1967) 172-186.

[49] M.M. Meerschaert, E. Nane, and P. Vellaisamy. Distributed-order fractional diffusions on bounded domains, J. Math. Anal. Appl. 379 (2011) 216-228.

[50] R. Metzler, J.-H. Jeon, A. G. Cherstvy, E. Barkai, Anomalous diffusion models and their properties: nonstationarity, non-ergodicity, and ageing at the centenary of single particle tracking, Phys. Chem. Chem. Phys. 16 (2014) 24128-24164.

[51] R. Metzler and J. Klafter, The random walk's guide to anomalous diffusion: a fractional dynamics approach, Phys. Rep. 339 (2000) 1-77.

[52] R. Metzler, J. Klafter, The restaurant at the end of the random walk: recent developments in the description of anomalous transport by fractional dynamics, J. Phys. A. Math. Gen. 37 (2004) R161-R208.

[53] E.W. Montroll and G. H. Weiss, Random Walks on Lattices. III. Calculation of First-Passage Times with Application to Exciton Trapping on Photosynthetic Units, J. Math. Phys. 10 (1969) 753-765.

[54] M.L. Morgado, M. Rebelo, Numerical approximation of distributed order reaction-diffusion equations, J. Comput. Appl. Math. 275 (2015) 216-227.

[55] K. Pearson, The Problem of the Random Walk, Nature 72 (1905) 294.

[56] L.F. Richardson, Atmospheric diffusion shown on a distant neighbour graph, Proc. Roy. Soc. A 110 (1926) 709-737.

[57] B. Ross, A brief history and exposition of the fundamental theory of fractional calculus, Springer (1975) 1-36.

[58] T. Sandev, A.V. Chechkin, N. Korabel, H. Kantz, I.M. Sokolov, R. Metzler, Distributed-order diffusion equations and multifractality: Models and solutions, Phys Rev E Stat Nonlin Soft Matter Phys. 92 (2015) 042117.

[59] H. Scher and E. W. Montroll, Anomalous transit-time dispersion in amorphous solids, Phys. Rev. B 12 (1975) 2455.

[60] W.R. Schneider and W. Wyss, Fractional diffusion and wave equations, J. Math. Phys. 30 (1989) $134-144$.

[61] M. von Smoluchowski, Zur kinetischen Theorie der Brownschen Molekularbewegung und der Suspensionen, Ann. Phys. 21 (1906) 756-780. 
[62] A. Sommariva, M. Vianello,R. Zanovello, Adaptive bivariate Chebyshev approximation, Numerical Algorithms 38 (120053) 79-94.

[63] X. Wang, F. Liu, X. Chen, Novel Second-Order Accurate Implicit Numerical Methods for the Riesz Space Distributed-Order Advection-Dispersion Equations, Adv. Math. Phys. 2015 (2015).

[64] A. Weron, M. Magdziarz, Generalization of the Khinchin theorem to Lévy flights, Phys. Rev. Lett. 105 (2010) 260-603.

[65] H. Ye, F. Liu, V. Anh, I. Turner, Numerical analysis for the time distributed-order and Riesz space fractional diffusions on bounded domains, IMA J. Appl. Math. 80 (2015) 825-838.

[66] H. Ye, F. Liu, V. Anh., Compact difference scheme for distributed-order time-fractional diffusion-wave equation on bounded domains, J.of Computat. Phys. 298 (2015) 652-660. 